\title{
Transition Navigator Intervention improves transition readiness to adult care and addresses barriers for youth with Sickle Cell Disease
}

\author{
Deepa Manwani ${ }^{1}$, Maya Doyle ${ }^{2}$, Lynn Davidson ${ }^{3}$, Makeda Mallea ${ }^{1}$, Ellen Silver ${ }^{4}$, Jenai \\ Jackson $^{4}$, Karen Ireland ${ }^{5}$, Rosy Chhabra ${ }^{6}$, Kerry Morrone ${ }^{7}$, Caterina Minniti ${ }^{4}$, Deepa \\ Rastogi $^{8}$, Ruth Stein ${ }^{6}$, Suzette Oyeku ${ }^{7}$, and Laurie Bauman ${ }^{6}$ \\ ${ }^{1}$ Children's Hospital at Montefiore \\ ${ }^{2}$ Quinnipiac University \\ ${ }^{3}$ Albert Einstein college of Medicine \\ ${ }^{4}$ Yeshiva University Albert Einstein College of Medicine \\ ${ }^{5}$ Childrenś Hospital at Montefiore \\ ${ }^{6}$ Albert Einstein College of Medicine \\ ${ }^{7}$ The Children's Hospital at Montefiore \\ ${ }^{8}$ Children's National Hopsital
}

October 12, 2020

\begin{abstract}
Background Adolescents and young adults (AYA) with Sickle Cell Disease (SCD) experience especially high rates of acute care utilization and increased morbidity, due in part to disease exacerbation and in part to the developmental challenges of adolescence. It is at this very high-risk time that they also face the need to transition their care to adult services, further adding to the heightened morbidity and acute care utilization, if poorly coordinated. Previous research supports the effectiveness of patient navigators to assist those with complex health conditions and healthcare needs, and has also identified the utility of a transition navigator for youth with special health care needs. Methods We developed a protocolized transition navigator intervention that used ecological assessment and motivational interviewing to assess transition readiness, identify goals, and remove barriers to transition, as well as to provide disease and pain management education and skills to AYAs with SCD. We assessed feasibility, acceptability and short-term efficacy in $n=60$ youth aged 17-20 with SCD. Findings Participation in the TN program was associated with significant improvement in transition readiness, disease knowledge, and confidence in disease and pain management for youth with SCD Conclusion The TN intervention was acceptable to youth with SCD and feasible to implement at an urban academic medical center and addressed most of the barriers to transition identified by the youth. Longer-term assessment is needed to determine if the transition navigator intervention improved successful transfer to and retention in adult care and reduced morbidity and ED reliance over time.
\end{abstract}

\section{Keywords (3-6)}

healthcare transition, navigator, sickle cell disease, adolescent, young adult

Introduction

Sickle Cell Disease (SCD) is a chronic illness characterized by vaso-occlusive complications leading to unpredictable episodes of pain, cumulative organ damage ${ }^{1,2}$ and high rates of health care utilization. ${ }^{3,4}$ Adolescence and young adulthood is a particularly high-risk period for people with SCD, and is characterized by increased health care utilization and death. ${ }^{5-7}$ Thirty-day readmission rates are used as a quality metric for a variety of chronic diseases: $41 \%$ of patients ages 18-30 with SCD are readmitted within 30 days, 
compared to $3.4 \%$ for asthma and $12 \%$ for pneumonia. ${ }^{5,8}$ A benchmark study ${ }^{5}$ reported highest rates of emergency department (ED) encounters and admission rates among SCD individuals 18-30 years of age. Factors in this vulnerable period associated with poorer outcomes include the cumulative effects of chronic illness; ${ }^{9}$ psychological changes during adolescence resulting in decreased adherence with medication; ${ }^{10}$ and worsening burden of neurocognitive deficits with advancing age..$^{9,11}$

A strong patient-provider partnership can be an important protective factor and reduce risk in this age group, but few young people with SCD transition smoothly to an adult care provider. Barriers to successful transition from pediatric to adult care have been identified previously ${ }^{12-16}$ and include lack of an identified staff member responsible for transition planning, unwillingness of families and pediatric providers to separate,${ }^{15}$ pediatricians' concerns that unstable patients will not receive the quality of care they need, ${ }^{13-18}$ and difficulty finding an adult provider with the knowledge and skills to care for them. ${ }^{18-22}$ Lack of transition planning results in poor quality of care and low patient satisfaction. ${ }^{23}$

Several consensus papers over the past two decades ${ }^{13,24-26}$ have recommended steps to assure effective transition, including an individualized transition plan, a portable medical summary, counseling to improve patient knowledge and skills for self-management, and referral to an appropriate adult provider. However, across subspecialties and healthcare networks, few pediatric practices follow these recommendations consistently. ${ }^{27,} 28$

The role of a navigator or care coordinator who assists those with complex health conditions and healthcare needs has been demonstrated. ${ }^{29-32}$ A retrospective report on the use of a transition navigator (TN) for patients with SCD at a single institution in Canada reported increased self-reported medication adherence and decreased loss to follow up as compared to a cohort assessed prior to implementation of the program. ${ }^{31}$ Canada's publicly funded health care system differs vastly from the healthcare landscape in the US. Patient navigator programs are relatively more common in Canada and a recent environmental scan described 23 pediatric programs. ${ }^{33}$

This study built on these findings by developing a protocolized TN intervention intended to facilitate transition readiness and remove barriers to transition in youth with SCD aged 17-20. . The program manual identifies the number of sessions, session tasks and goals, and timing of the intervention (see Supplement 1). Here we describe feasibility (defined as success in recruiting eligible patients and retaining them throughout the intervention); acceptability (measured in a post-intervention survey), and impact on short term (interim) results on transition readiness, disease knowledge, success in addressing patient and system barriers to transition $\backslash$ sout.

Methods

This proof-of-concept single group pre-post study was approved by the Institutional Review Board of the Albert Einstein College of Medicine/Montefiore Medical Center. We enrolled 60 youth with SCD between May 2016 and July 2017. They completed questionnaires (described below) at baseline and 6-12 months later, after completion of the structured intervention

\section{Navigator Training and Role}

In designing the TN role for this study, an ecological assessment approach ${ }^{34-36}$ and a motivational interviewing style ${ }^{37,38}$ was adopted. The consensus statements on transition ${ }^{12,24}$ recommend areas of attention for clinicians; a typically under-addressed area is a focus on future goals. ${ }^{39}$ The TN training modules emphasized investigating, acknowledging, and supporting the future goals of AYAs with SCD. TN training included the consensus statements, online healthcare transition modules and continuing education ${ }^{40,}{ }^{41}$, related literature ${ }^{42}$, transition materials designed for patients and families by the institution and recommended local and national resources on the web $^{43}$ (see Supplemental 1). Educational materials specific to SCD inheritance, pathophysiology, treatment, complications, and self-management were also incorporated. The intervention could be implemented by a TN with a social work, nursing, or patient educator background.

\section{Intervention design}


The intervention aimed to improve patient knowledge and skills for disease self-management and to prepare for transfer to an adult care provider, with five components derived from GOT TRANSITION $\AA$ best practice recommendations: ${ }^{43}$

- An individualized transition plan

- A portable medical summary co-constructed by patient and TN, and reviewed by the pediatric hematology provider(s) prior to transition utilizing MyHealth Passport ${ }^{44}$

- Education about SCD and the skills needed for disease self-management, particularly addressing quality of care, adherence, and utilization of emergency services

- Education of family members about their role in transition while focusing intervention on AYAs

- Facilitating transfer to an adult provider (appointment-making, accompaniment, and post-appointment follow-up call).

As shown in the Process Map of Intervention (see Table 1), each youth had three planned interactions with TN over 6-12 months and a 3-month post-intervention follow-up call. Additional calls were permitted as needed. The first visit entailed an in-person protocolized TN transition assessment which included future plans for healthcare and mental health services; educational and vocational goals; housing and geographic relocations; changing decision-making abilities and rights; insurance coverage needs, changes, and rights; and current providers and support system (see Supplement 1). SCD specific knowledge tools were also introduced at this visit. Visits 2 and 3 addressed identified gaps in disease knowledge/ management and skills; development of a portable medical summary; and identification and contact with adult provider. Barriers to transition were reviewed at each visit, and a barriers checklist (see Supplement 1) was completed by the TN based on AYA self-report and TN observation. The TN completed the sessions at mutually agreed upon times and there was flexibility for each visit to be broken up into several in-person or phone visits based on patient preference. The TN kept detailed records of the dates and time spent for each interaction and whether they occurred in person or by phone. The TN also met weekly for case review with the SCD clinical lead as well as with the entire SCD clinical team consisting of the physicians, nurse practitioners, and social worker.

\section{Recruitment}

Clinic rosters of youth in the SCD hematology clinic were assessed for the following eligibility criteria: (1) aged 17-20 inclusive; (2) confirmed diagnoses of SCD (SS, SC, SBeta Thalassemia genotypes); (3) cared for in the subspecialty Pediatric Hematology service; (4) spoke English. Eligible patients were telephoned and invited to participate in the study. Those who were interested met the study coordinator at their next medically indicated clinic visit; those 18 and older provided written informed consent; those who were aged 17 provided assent and their parents provided permission.

\section{Survey Instruments}

Youth completed survey instruments at enrollment (T1) and program completion (T2). A list of the survey measures, their purpose, source, reliability, and validity are detailed in Table 2 . These instruments included both validated measures as well as process measures adapted from tools recommended by Got Transition $\mathbb{B}$ and previous assessments of provider transition practice at the institution ${ }^{27}$, ${ }^{43}$ These measures assessed transition readiness, health literacy, SCD knowledge, confidence in managing SCD and pain management, SCD control, self-reported health care utilization, patient satisfaction with transition and satisfaction with the TN intervention. All data was entered into REDCap ${ }^{45}$ by the research coordinator.

\section{Data Analysis}

Summary statistics (frequencies, percentages) were computed to describe participant characteristics and their contacts with the TN during the intervention. Pre/post scores on measures of transition readiness, SCD knowledge, confidence in managing SCD, medication adherence and pain management were compared using paired t-tests and the associations of patient-TN contacts with post-test measures were analyzed using correlations. Pre/post ratings of participant's experience of interaction with their healthcare provider regarding transition were compared using McNemar tests for paired analysis. Intake assessments were completed 
on paper by the TN; insurance/SSI status and items related to education and career goals were hand-coded from this assessment by two members of the research team. Barriers to transition were identified through patient self-report and TN observation, and separately documented on the barriers checklist (see supplement 1); responses by participants and $\mathrm{TN}$ were compared using McNemar tests.

\section{Findings}

\section{Demographics}

In total, 62 AYAs met study criteria; 60 were recruited into the study and 2 refused participation. Of the 60 enrolled participants, 56 completed the intervention, 1 was incarcerated during intervention timeframe and 3 dropped out. The analysis was completed on the 56 who completed both study questionnaires. Demographics, including gender, diagnosis, race, age, insurance and SSI status, and participant-reported educational status and vocational goals are shown in Table 3 . Of note, $85.9 \%$ reported that they were either in college, applying, or planning to go to college and $42.1 \%$ were actually enrolled in college.

At intake, $48 \%$ of participants reported needing help reading hospital materials sometimes/often/always, $27 \%$ felt not at all/a little bit/somewhat confident in completing medical forms, and $14 \%$ reported sometimes/often/always having problems learning about their medical condition because of difficulty understanding written information.

Short-term (interim) outcomes: Transition readiness, disease self-management scales and barriers to transition

Pre/post scores for the TRAQ, SCD Knowledge, Confidence Managing SCD, Pain Management and AMBS scales are shown in Table 4. There was significant improvement in total TRAQ scores on 3 of the 5 TRAQ subscales; the two subscales that did not show significant change were already high at baseline. SCD knowledge also improved significantly. In addition to the SCD knowledge questionnaire, patient's self-identification of SCD genotype was assessed at baseline and at exit. At enrollment $80 \%$ knew their genotypes correctly, with 3 giving wrong answers and 8 saying "don't know." Of the 11 that did not report genotype correctly at enrollment, 9 were able to do so at exit. In the post-test survey, $95 \%$ correctly reported their genotypes. Scores on both confidence in disease management and use of disease/pain management skills subscales also improved significantly, while barriers to medication adherence decreased significantly from baseline to exit (Table 4).

As part of the TN intake in Session 1, participants were asked to identify transition barriers they felt or experienced, and the TN recorded these as well as barriers she observed throughout the intervention period (Table 5). Significant differences between participants' and TN's identification of barriers were seen in several areas: difficulty identifying a provider, lack of appointment availability, lack of insurance, and provider delay in transition planning. Top concerns of the TN were patient's relocation to college and parent/patient's reluctance to transition due to difficulty "letting go." Patients' top concerns were difficulty finding a new provider/relocation, identifying a new provider and relocation for college. Of these participants, $42 \%$ were in college at post-test and an additional $44 \%(25 / 57)$ were planning to or applying to go to college.

Participant acceptability and satisfaction with the intervention

Participants were asked to assess their experience and understanding of the transition process and interactions with healthcare team in both the pre- and post-intervention surveys (see Table 6). While changes seen in scores from pre- to post-intervention all indicated significant improvement, the most striking change was on the item "Know how you will become insured as an adult" (pre $=16.1 \%$ vs post $=78.6 \%, \mathrm{p}<.0001$ ). Youth expressed high levels of satisfaction with the program with $>98 \%$ stating that the TN had explained the program, they understood the program content, and they had sufficient time to ask questions at sessions. Of participants that responded to the query, 52 out of $54(96.2 \%)$ stated that they preferred to stay within the current hospital system.

Feasibility of the intervention 
Feasibility is defined as the extent to which a new treatment or innovation can be successfully implemented in a given agency or setting. ${ }^{46}$ We assessed feasibility in terms of success in participant recruitment, retention, and participation rates. We reported earlier that we successfully recruited 60 of 62 eligible patients and completed the intervention in 56 of the 60 enrolled. In addition, we were able to complete all components of the intervention by telephone or in person within the 12-month intervention window. We examined associations between post-test measures and amount of contact between patients and the TN, but only a few significant associations were noted. Having more in-person visits $(\mathrm{r}=.32 ; \mathrm{p}=.015)$ and having fewer telephone contacts $(\mathrm{r}=-.38 ; \mathrm{p}=.003)$ both were correlated with higher post-test TRAQ appointment keeping scores, but contact time was not associated with any other TRAQ subscales, SCD knowledge, or pain management scores at post-test. As might be expected, more contact time was required by those with less confidence in managing $\operatorname{SCD}(\mathrm{r}=-.29 ; \mathrm{p}=.03)$ and those with higher disease frustration $(\mathrm{r}=.31 ; \mathrm{p}=.02)$ and medication barriers $(\mathrm{r}=.28 ; \mathrm{p}=.04)$ scores on the AMBS.

\section{Discussion}

This study demonstrates that the use of an intensive TN intervention in a US urban academic center subspecialty practice is feasible, acceptable to participants, and effective in improving transition readiness and disease knowledge, and reducing patient and systems barriers to transition. Several "lessons learned" were identified by the TN and the team in implementing a transition intervention for AYAs with SCD.

AYAs with SCD acknowledge the importance of transfer from pediatric to adult care but often demonstrate poor readiness for this important care transition. ${ }^{47}$ Thus, this TN intervention focused on readiness and barriers, working with the participants to address those concerns. Providers were encouraged to be aware of the barriers in transition planning, ${ }^{28}$ especially since the roles of the pediatrician and the adult physician and the ways in which they interact with their patients are quite different. ${ }^{48}$ Many of the barriers identified by our participants indicated lack of familiarity with negotiating the healthcare system or insurance, rather than actual systemic barriers, and these were readily addressed by TN.

The high rate of concern by the TN and participants related to college attendance and health care access was realistic, and reflected a confluence of barriers: the logistics of transfer to adult care in a setting unfamiliar to both the TN and youth, several insurmountable out of state insurance coverage issues, and the demands on the youth to master several novel tasks simultaneously. The AYAs in our sample had high rates of attending or planning to attend college. As of 2019, in the Bronx, 37.3\% of 18-24 year old youth were enrolled in or graduated from college. ${ }^{49}$ Thus, the AYAs in our sample reported similar rates of attending college compared to the general Bronx population. It is noteworthy that despite their college plans, the AYAs in our study reported significant health literacy concerns. The experience with the college bound youth in our program led to changes in pediatric hematology service policies - to implement more intensive case management and coordination of care with a local provider near the college to assure access to urgent and emergency services as well as pain management.

There are several ways the intervention could be improved. The time of emerging adulthood ${ }^{50}$ and healthcare transition is a time of immense change in many aspects of life: education, vocation, living arrangements, relationships, financial independence, and legal decision-making. ${ }^{39}$ Participants were recruited for this study between the age of 17 and 20, as they neared time for transfer to adult care, which was based on restrictions imposed by the funding available for the program. However, the consensus recommendation is to start the transition process earlier, at 12 or $13 .^{13,24,25}$ This recommendation reflects the need for increased age-appropriate disease education, skills acquisition and practice, and increased responsibility for self-care as youth navigate the developmental challenges of increasing independence, peer relationships, and future planning. A longer period of education about self-management skills and disease knowledge may be essential to significantly impact ED reliance and appropriate health care utilization. As health care utilization is expected to increase over time in SCD, other investigators have reported stabilization as a potentially positive effect. ${ }^{31}$ Without a comparison group we could not assess the efficacy of the TN intervention on health care use or morbidity. Starting earlier would allow youth to spend more time with the TN and to build greater rapport and trust, which the $\mathrm{TN}$ reported to be a key factor for the success of the intervention. On a related 
note, for a study population of predominantly Black/African-American and Hispanic AYA's with SCD ${ }^{51}$ it seems pertinent to note also that the TN was a woman of color. In addition, the TN noted the participants' desires for peer support, suggesting the need for/appeal of transition mentors (slightly older patients who have recently successfully transitioned), and/or a transition buddy system (providing a shared experience).

As healthcare systems see greater use of and payment for telehealth or video platforms in the wake of COVID, such platforms may be particularly useful both for engagement and for follow-up needed to enhance transition from pediatric to adult-oriented care. The most recent position paper from the Society for Adolescent Health and Medicine includes an endorsement of greater integration of technology in healthcare transitioning, including the electronic health record and telehealth. ${ }^{26}$ Expanding the TN intervention in this way could be time- and resource-saving and allow greater choice and flexibility for providers, families, and patients, particularly for AYA's acclimated to technology use. Although an initial in-person TN assessment may help in creating a working relationship or sharing educational materials, shorter follow-up calls could be completed and documented without an in-person appointment. In addition, telemedicine for transition preparation and education may be a more acceptable time-saving format for AYAs as well. While our intervention was not designed to assess telehealth, the data kept by the TN indicated that visits by phone were often successful when in-person visits could not be accommodated into a participant's or provider's schedule, and these calls allowed for both education and case management activities that might otherwise have been missed.

\section{Limitations}

This study was a single-group pre-post proof of concept study. The lack of a comparison group is a weakness although the magnitude of the improvements seen following the intervention provides significant support for conducting a larger randomized trial. Because the youth did not transfer to adult care during the study period, the impact on this important metric will need to be assessed in future studies as well. Some measures of program outcome were designed by the study team (e.g., SCD knowledge) and need further psychometric assessment. The study originally planned to enroll a second group of youth that would include 60 AYA's with persistent asthma cared for in a primary care clinic, along with the 60 AYAs with SCD we did recruit. However, barriers arose early in our attempt to utilize the primary care setting including determining acuity of persistent asthma to fit recruitment criteria, finding space to meet with patients, and integrating TN into the existing primary care team. For this reason, the efforts of the TN reported here focus on AYA with SCD who were seen in the subspecialty Pediatric Hematology service. Other limitations included lack of sustainability for the program based mainly on lack of a billing option, and reliance on a single individual TN rather than making transition planning and care part of the culture of the entire clinical team. The impact of the COVID-19 pandemic on our medical institution also interrupted ongoing transition planning and availability of adult providers.

\section{Conclusions}

Our findings demonstrate that participation in an intensive TN intervention targeting adolescents and young adults 17-20 years old with SCD over 6-12 months was associated with significant improvement in transition readiness, disease knowledge, and confidence in disease and pain management. The intervention was acceptable to youth and feasible to implement at an urban academic medical center. Further testing of the feasibility of adapting this TN intervention for telehealth or video implementation is warranted, as this could allow for more efficient delivery and for increased impact to a larger patient population as well. Longer-term assessment is needed to determine whether a transition navigator intervention improves successful transfer to and retention in adult care, and if it reduces morbidity, ED reliance and hospitalization over time.

Authorship: DM, LB, MD, EJS designed the study, analyzed the data and wrote the manuscript. LD designed the study, analyzed the data and revised the manuscript RC, DR, RS, SO designed the study, analyzed the data and reviewed the manuscript. DM oversaw the study conduct and data collection. JJ analyzed the data and wrote the manuscript. MM, KI, KM, CM conducted the study, collected data and reviewed the manuscript. EJS performed statistical analyses. 
Conflict of interest statement: The authors declare no competing financial conflicts.

Data available on request from the authors

References

1. Manwani, D.; Frenette, P. S., Vaso-occlusion in sickle cell disease: pathophysiology and novel targeted therapies. Blood 2013, 122 (24), 3892-8.

2. Kato, G. J.; Piel, F. B.; Reid, C. D.; Gaston, M. H.; Ohene-Frempong, K.; Krishnamurti, L.; Smith, W. R.; Panepinto, J. A.; Weatherall, D. J.; Costa, F. F.; Vichinsky, E. P., Sickle cell disease. Nat Rev Dis Primers 2018, 4 (1), 18010.

3. Shankar, S. M.; Arbogast, P. G.; Mitchel, E.; Cooper, W. O.; Wang, W. C.; Griffin, M. R., Medical care utilization and mortality in sickle cell disease: a population-based study. Am J Hematol2005, 80 (4), 262-70.

4. Steiner, C. A.; Miller, J. L., Sickle Cell Disease Patients in U.S. Hospitals, 2004: Statistical Brief \#21. In Healthcare Cost and Utilization Project (HCUP) Statistical Briefs, Rockville (MD), 2006.

5. Brousseau, D. C.; Owens, P. L.; Mosso, A. L.; Panepinto, J. A.; Steiner, C. A., Acute care utilization and rehospitalizations for sickle cell disease. JAMA 2010, 303 (13), 1288-94.

6. Paulukonis, S. T.; Eckman, J. R.; Snyder, A. B.; Hagar, W.; Feuchtbaum, L. B.; Zhou, M.; Grant, A. M.; Hulihan, M. M., Defining sickle cell disease mortality using a population-based surveillance system, 2004 through 2008. Public Health Reports 2016,131 (2), 367-375.

7. Hemker, B. G.; Brousseau, D. C.; Yan, K.; Hoffmann, R. G.; Panepinto, J. A., When children with sicklecell disease become adults: lack of outpatient care leads to increased use of the emergency department. $A m$ J Hematol 2011, 86 (10), 863-5.

8. Berry, J. G.; Hall, D. E.; Kuo, D. Z.; Cohen, E.; Agrawal, R.; Feudtner, C.; Hall, M.; Kueser, J.; Kaplan, W.; Neff, J., Hospital utilization and characteristics of patients experiencing recurrent readmissions within children's hospitals. JAMA 2011,305 (7), 682-90.

9. Kayle, M.; Docherty, S. L.; Sloane, R.; Tanabe, P.; Maslow, G.; Pan, W.; Shah, N., Transition to adult care in sickle cell disease: A longitudinal study of clinical characteristics and disease severity.Pediatr Blood Cancer 2019, 66 (1), e27463.

10. Green, N. S.; Manwani, D.; Qureshi, M.; Ireland, K.; Sinha, A.; Smaldone, A. M., Decreased fetal hemoglobin over time among youth with sickle cell disease on hydroxyurea is associated with higher urgent hospital use. Pediatric blood \&3 cancer 2016, 63(12), 2146-2153.

11. DeBaun, M. R.; Telfair, J., Transition and sickle cell disease.Pediatrics 2012, 130 (5), 926-35.

12. American Academy of Pediatrics; American Academy of Family Physicians; American College of, P.; Transitions Clinical Report Authoring Group; Cooley, W. C.; Sagerman, P. J., Supporting the health care transition from adolescence to adulthood in the medical home.Pediatrics 2011, 128 (1), 182-200.

13. White, P. H.; Cooley, W. C.; Transitions Clinical Report Authoring, G.; American Academy Of, P.; American Academy Of Family, P.; American College Of, P., Supporting the Health Care Transition From Adolescence to Adulthood in the Medical Home. Pediatrics 2018,142 (5).

14. Porter, J. S.; Wesley, K. M.; Zhao, M. S.; Rupff, R. J.; Hankins, J. S., Pediatric to Adult Care Transition: Perspectives of Young Adults With Sickle Cell Disease. J Pediatr Psychol 2017, 42 (9), 1016-1027.

15. Porter, J. S.; Graff, J. C.; Lopez, A. D.; Hankins, J. S., Transition from pediatric to adult care in sickle cell disease: perspectives on the family role. J Pediatr Nurs 2014,29 (2), 158-67.

16. Bemrich-Stolz, C. J.; Halanych, J. H.; Howard, T. H.; Hilliard, L. M.; Lebensburger, J. D., Exploring Adult Care Experiences and Barriers to Transition in Adult Patients with Sickle Cell Disease. Int $J$ Hematol 
Ther 2015, 1 (1).

17. McManus, M. A.; Fox, H.; O'Connor, K.; Chapman, T.; MacKinnon, J.,Pediatric perspectives and practices on transitioning adolescents with special needs to adult health care. National Alliance to Advance Adolescent Health Washington DC: 2008.

18. McManus, M. A.; Pollack, L. R.; Cooley, W. C.; McAllister, J. W.; Lotstein, D.; Strickland, B.; Mann, M. Y., Current Status of Transition Preparation Among Youth With Special Needs in the United States.Pediatrics 2013, 131 (6), 1090-1097.

19. Okumura, M. J.; Kerr, E. A.; Cabana, M. D.; Davis, M. M.; Demonner, S.; Heisler, M., Physician views on barriers to primary care for young adults with childhood-onset chronic disease. Pediatrics 2010, 125 (4), e748-e754.

20. Sobota, A.; Neufeld, E. J.; Sprinz, P.; Heeney, M. M., Transition from pediatric to adult care for sickle cell disease: results of a survey of pediatric providers. Am J Hematol 2011,86 (6), 512-5.

21. Telfair, J.; Ehiri, J. E.; Loosier, P. S.; Baskin, M. L., Transition to adult care for adolescents with sickle cell disease: results of a national survey. Int $J$ Adolesc Med Health 2004,16 (1), 47-64.

22. Lebensburger, J. D.; Bemrich-Stolz, C. J.; Howard, T. H., Barriers in transition from pediatrics to adult medicine in sickle cell anemia.J Blood Med 2012, 3 , 105-12.

23. Lanzkron, S.; Carroll, C. P.; Haywood, C., Jr., Mortality rates and age at death from sickle cell disease: U.S., 1979-2005. Public Health Rep 2013, 128 (2), 110-6.

24. American Academy of Pediatrics; American Academy of Family Physicians; American College of Physicians; American Society of Internal Medicine, A consensus statement on health care transitions for young adults with special health care needs. Pediatrics 2002,110 (6), 1304-6.

25. American Academy of, P.; American Academy of Family, P.; American College of, P.; Transitions Clinical Report Authoring, G.; Cooley, W. C.; Sagerman, P. J., Supporting the health care transition from adolescence to adulthood in the medical home. Pediatrics 2011, 128 (1), 182-200.

26. Society for Adolescent, H.; Medicine, Transition to Adulthood for Youth With Chronic Conditions and Special Health Care Needs. J Adolesc Health 2020, 66 (5), 631-634.

27. Davidson, L. F.; Doyle, M.; Silver, E. J., Multidisciplinary Support for Healthcare Transitioning Across an Urban Healthcare Network. J Pediatr Nurs 2015, 30 (5), 677-83.

28. Davidson, L. F.; Chhabra, R.; Cohen, H. W.; Lechuga, C.; Diaz, P.; Racine, A., Pediatricians Transitioning Practices, Youth With Special Health Care Needs in New York State. Clin Pediatr (Phila)2015, 54 (11), 1051-8.

29. Calhoun, E. A.; Esparza, A., Patient Navigation: Overcoming Barriers to Care . Springer: 2017.

30. Esparza, A.; Calhoun, E., Measuring the impact and potential of patient navigation: proposed common metrics and beyond. Cancer2011, 117 (15 Suppl), 3537-8.

31. Allemang, B.; Allan, K.; Johnson, C.; Cheong, M.; Cheung, P.; Odame, I.; Ward, R.; Williams, S.; Mukerji, G.; Kuo, K. H. M., Impact of a transition program with navigator on loss to follow-up, medication adherence, and appointment attendance in hemoglobinopathies.Pediatr Blood Cancer 2019, 66 (8), e27781.

32. Mackie, A. S.; Rempel, G. R.; Kovacs, A. H.; Kaufman, M.; Rankin, K. N.; Jelen, A.; Manlhiot, C.; Anthony, S. J.; Magill-Evans, J.; Nicholas, D.; Sananes, R.; Oechslin, E.; Dragieva, D.; Mustafa, S.; Williams, E.; Schuh, M.; McCrindle, B. W., A cluster randomized trial of a transition intervention for adolescents with congenital heart disease: rationale and design of the CHAPTER 2 study. BMC cardiovascular disorders 2016 , 16,127 . 
33. Luke, A.; Doucet, S.; Azar, R., Paediatric patient navigation models of care in Canada: An environmental scan. Paediatr Child Health 2018, 23 (3), e46-e55.

34. Champaloux, S. W.; Young, D. R., Childhood chronic health conditions and educational attainment: a social ecological approach. J Adolesc Health 2015, 56 (1), 98-105.

35. Griffin, A.; Gilleland, J.; Johnson, A.; Cummings, L.; New, T.; Brailey, T.; Eckman, J.; Osunkwo, I., Applying a developmental-ecological framework to sickle cell disease transition. Clinical Practice in Pediatric Psychology 2013, 1(3), 250.

36. Wang, G.; McGrath, B. B.; Watts, C., Health Care Transitions Among Youth With Disabilities or Special Health Care Needs: An Ecological Approach. Journal of Pediatric Nursing 2010, 25(6), 505-550.

37. Erickson, S. J.; Gerstle, M.; Feldstein, S. W., Brief interventions and motivational interviewing with children, adolescents, and their parents in pediatric health care settings: a review. Archives of Pediatrics $\mathcal{E}^{3}$ Adolescent Medicine 2005, 159 (12), 1173-1180.

38. Naar-King, S.; Suarez, M., Motivational interviewing with adolescents and young adults . Guilford Press: 2011.

39. Davidson, L. F.; Doyle, M.; Silver, E. J., Discussing Future Goals and Legal Aspects of Health Care: Essential Steps in Transitioning Youth to Adult-Oriented Care. Clin Pediatr (Phila) 2017,56 (10), 902-908.

40. FloridaHATS Health Care Transition Training for Health Care Professionals. https://www.floridahats.org/education-training-for-health-care-professionals/.

41. HSC Healthcare System Health Care Transition for Adolescents and Young Adults- An Online Video CME/CEU Series. https://www.hscsnlearning.org/transition/index.php.

42. Wood, D. L.; Sawicki, G. S.; Miller, M. D.; Smotherman, C.; Lukens-Bull, K.; Livingood, W. C.; Ferris, M.; Kraemer, D. F., The Transition Readiness Assessment Questionnaire (TRAQ): its factor structure, reliability, and validity. Acad Pediatr 2014,14 (4), 415-22.

43. GotTransition (B) National Health Care Transition Center Six Core Elements of Health Care Transition. https://www.gottransition.org/six-core-elements/.

44. SickKids My Health Passport. http://www.sickkids.ca/good2go/what-we-do/myhealthpassport/index.html.

45. Harris, P. A.; Taylor, R.; Thielke, R.; Payne, J.; Gonzalez, N.; Conde, J. G., Research electronic data capture (REDCap) - a metadata-driven methodology and workflow process for providing translational research informatics support. Journal of biomedical informatics 2009, 42 (2), 377-381.

46. Karsh, B. T., Beyond usability: designing effective technology implementation systems to promote patient safety. Qual Saf Health Care 2004, 13 (5), 388-94.

47. McPherson, M.; Thaniel, L.; Minniti, C. P., Transition of patients with sickle cell disease from pediatric to adult care: Assessing patient readiness. Pediatr Blood Cancer 2009, 52 (7), 838-41.

48. Stein, R.; Perrin, J.; Iezzoni, L., Access and medical support for youth and young adults with chronic health conditions and disabilities. In Launching Into Adulthood: An Integrated Response to Support Transition of Youth With Chronic Health Conditions and Disabilities. : , Lollar, D. J., Ed. Paul H Brookes Publishing Co: Baltimore, MD, 2010; pp 77-102.

49. Towncharts.com, Bronx, New York Education Data from the 2019 American Community Survey. In Towncharts.com - United States data powerfully illustrated and interactive. , 2019.

50. Arnett, J. J., Emerging adulthood: The winding road from the late teens through the twenties . Oxford University Press: 2014. 
51. Rouse, C., Uncertain suffering: racial health care disparities and sickle cell disease. Univ of California Press: 2009.

52. Chew, L. D.; Griffin, J. M.; Partin, M. R.; Noorbaloochi, S.; Grill, J. P.; Snyder, A.; Bradley, K. A.; Nugent, S. M.; Baines, A. D.; Vanryn, M., Validation of screening questions for limited health literacy in a large VA outpatient population. J Gen Intern Med 2008,23 (5), 561-6.

53. Wallace, L. S.; Rogers, E. S.; Roskos, S. E.; Holiday, D. B.; Weiss, B. D., Brief report: screening items to identify patients with limited health literacy skills. J Gen Intern Med 2006, 21(8), 874-7.

54. Sawicki, G. S.; Lukens-Bull, K.; Yin, X.; Demars, N.; Huang, I.-C.; Livingood, W.; Reiss, J.; Wood, D., Measuring the transition readiness of youth with special healthcare needs: validation of the TRAQTransition Readiness Assessment Questionnaire. Journal of pediatric psychology 2009 , jsp128.

55. Prochaska, J.; DiClemente, C.; Norcross, J., Handbook of eclectic psychotherapy. New York: BrunnerMazel 1986 .

56. Rastogi, D.; Madhok, N.; Kipperman, S., Caregiver Asthma Knowledge, Aptitude, and Practice in High Healthcare Utilizing Children: Effect of an Educational Intervention. Pediatr Allergy Immunol Pulmonol2013, 26 (3), 128-139.

57. Nathan, R. A.; Sorkness, C. A.; Kosinski, M.; Schatz, M.; Li, J. T.; Marcus, P.; Murray, J. J.; Pendergraft, T. B., Development of the asthma control test: a survey for assessing asthma control. J Allergy Clin Immunol 2004, 113 (1), 59-65.

58. Simons, L. E.; Blount, R. L., Identifying barriers to medication adherence in adolescent transplant recipients. J Pediatr Psychol2007, 32 (7), 831-44.

\section{Hosted file}

Table 1.pdf available at https://authorea.com/users/363081/articles/486204-transitionnavigator-intervention-improves-transition-readiness-to-adult-care-and-addressesbarriers-for-youth-with-sickle-cell-disease

\section{Hosted file}

Table 2.pdf available at https://authorea.com/users/363081/articles/486204-transitionnavigator-intervention-improves-transition-readiness-to-adult-care-and-addressesbarriers-for-youth-with-sickle-cell-disease

\section{Hosted file}

Table 3.pdf available at https://authorea.com/users/363081/articles/486204-transitionnavigator-intervention-improves-transition-readiness-to-adult-care-and-addressesbarriers-for-youth-with-sickle-cell-disease

\section{Hosted file}

Table 4.pdf available at https://authorea.com/users/363081/articles/486204-transitionnavigator-intervention-improves-transition-readiness-to-adult-care-and-addressesbarriers-for-youth-with-sickle-cell-disease

\section{Hosted file}

Table 5.pdf available at https://authorea.com/users/363081/articles/486204-transitionnavigator-intervention-improves-transition-readiness-to-adult-care-and-addressesbarriers-for-youth-with-sickle-cell-disease

\section{Hosted file}


Table 6.pdf available at https://authorea.com/users/363081/articles/486204-transitionnavigator-intervention-improves-transition-readiness-to-adult-care-and-addressesbarriers-for-youth-with-sickle-cell-disease 